\title{
MEAN VALUES OF DERIVATIVES OF L-FUNCTIONS IN FUNCTION FIELDS: III
}

\author{
JULIO ANDRADE
}

\begin{abstract}
In this series of papers, we explore moments of derivatives of $L$-functions in function fields using classical analytic techniques such as character sums and approximate functional equation. The present paper is concerned with the study of mean values of derivatives of quadratic Dirichlet $L$-functions over function fields when the average is taken over monic and irreducible polynomials $P$ in $\mathbb{F}_{q}[T]$. When the cardinality $q$ of the ground field is fixed and the degree of $P$ gets large we obtain asymptotic formulas for the first moment of the first and the second derivative of this family of $L$-functions at the critical point. We also compute the full polynomial expansion in the asymptotic formulas for both mean values.
\end{abstract}

\section{INTRODUCTION}

This is the third paper of a series of papers devoted to studying mean values of derivatives of $L$-functions in function fields. In the first and second paper in this series $([4,1])$ we considered the first moment of derivatives of $L$-functions in function fields when the average is taken over an ensemble of square-free monic polynomials in $\mathbb{F}_{q}[T]$. In this paper we venture in compute mean values of derivatives of $L$-functions in function fields when the average is taken over monic and irreducible polynomials in $\mathbb{F}_{q}[T]$.

Hoffstein and Rosen [9] were the first to study mean values of $L$-functions in function fields. In their beautiful paper, they established several results concerning mean values of different families of $L$-functions in function fields. However, in their paper, they never consider mean values of $L$-functions associated to monic and irreducible polynomials. In this paper we investigate averages over monic and irreducible polynomials.

It is well-known that averages taken over primes are much harder to compute than averages over square-free numbers. The same principle also applies to the function field setting, where averages over monic irreducible polynomials are more difficult to handle than averages over square-free polynomials. This is mainly due to the fact that the primes (or monic irreducibles) are a

Date: April 10, 2017.

2010 Mathematics Subject Classification. Primary 11M38; Secondary 11M06, 11G20, $11 \mathrm{M} 50,14 \mathrm{G} 10$.

Key words and phrases. function fields, irreducible polynomials, hyperelliptic curves, derivatives of $L$-functions, moments of $L$-functions, quadratic Dirichlet $L$-functions, random matrix theory. 
thinner set when compared to the set of square-free numbers (or square-free polynomials).

In [3], Andrade and Keating computed the first and the second moment of quadratic Dirichlet $L$-functions in function fields when the average is taken over monic and irreducible polynomials in $\mathbb{F}_{q}[T]$. This paper can be thought as an extension of the papers [3, 9] where we handle the more intricate case of averages of quadratic Dirichlet $L$-functions, i.e., we handle averages over "primes" in this setting.

The study of derivatives of $L$-functions is an important problem in analytic number theory and has its roots in the work of Ingham [10, who established asymptotic formulas for the second moment of derivatives of the Riemann zeta function. Developments in the study of moments of derivatives of the Riemann zeta function were lead by Conrey [5] and Gonek [8]. In [6], by computing moments of the derivative of characteristic polynomials in the unitary group $U(N)$, Conrey, Rubinstein and Snaith formulated a general conjecture for the moments of derivatives of the Riemann zeta function. For a summarized account of the results in this paragraph, we ask the reader to refer [4, Section 1].

The main object of this paper is to study moments of derivatives of $L$ functions in the function field setting. In this note, we establish the first moment of the first and the second derivative of quadratic Dirichlet $L$-functions associated to monic irreducible polynomials in $\mathbb{F}_{q}[T]$. In future work (part 4 in this series) we establish more general mean values of derivatives of $L$-functions associated to monic and irreducible polynomials.

\section{MAIn TheOREMS}

The calculations in this paper will lead to the following theorems.

Theorem 2.1. Let $\mathbb{F}_{q}$ be a fixed finite field with $q$ odd. Then

$$
\begin{aligned}
\sum_{P \in \mathcal{P}_{2 g+1, q}} L^{\prime}\left(\frac{1}{2}, \chi_{P}\right) & =(\log q) \frac{|P|}{\log _{q}|P|}\left(\left[\frac{g-1}{2}\right]\left(1+\left[\frac{g-1}{2}\right]\right)\right. \\
& \left.-2 g\left(\left[\frac{g-1}{2}\right]+1\right)-\left[\frac{g}{2}\right]\left(\left[\frac{g}{2}\right]+1\right)\right) \\
& +O\left(|P|^{3 / 4}\left(\log _{q}|P|\right)\right) .
\end{aligned}
$$

Where $[x]$ indicates the integer part of $x,|P|=q^{2 g+1}$,

$$
\mathcal{P}_{2 g+1, q}=\left\{P \in \mathbb{F}_{q}[T], \text { monic and irreducible, and } \operatorname{deg}(P)=2 g+1\right\},
$$

and $L\left(s, \chi_{P}\right)$ is the quadratic Dirichlet L-function associated to $P$ where $\chi_{P}$ is the quadratic character defined by the Legendre symbol in $\mathbb{F}_{q}[T]$, i.e., 


$$
\chi_{P}(f)=\left(\frac{P}{f}\right) .
$$

Using that $2 g+1=\log _{q}|P|$ the next result follows as a simple corollary of Theorem 2.1 .

Corollary 2.1. Let $\mathbb{F}_{q}$ be a fixed finite field of odd cardinality $q$. Using the same notation as in the theorem we have,

$$
\sum_{P \in \mathcal{P}_{2 g+1, q}} L^{\prime}\left(\frac{1}{2}, \chi_{P}\right) \sim-\frac{1}{4} \log (q)|P|\left(\log _{q}|P|\right),
$$

as $g \rightarrow \infty$.

The next result is more involving and it is about the second derivative.

Theorem 2.2. Let $\mathbb{F}_{q}$ be a fixed finite field with $q$ odd. Using the same notation as in the theorem [2.1 we have that

$$
\begin{aligned}
& \sum_{P \in \mathcal{P}_{2 g+1, q}} L^{\prime \prime}\left(\frac{1}{2}, \chi_{P}\right)=\frac{2}{3}(\log (q))^{2} \frac{|P|}{\log _{q}|P|}\left(\left[\frac{g}{2}\right]\left(1+\left[\frac{g}{2}\right]\right)\left(1+2\left[\frac{g}{2}\right]\right)\right. \\
& \left.+\left(\left(1+\left[\frac{g-1}{2}\right]\right)\left(6 g^{2}+\left[\frac{g-1}{2}\right]-6 g\left[\frac{g-1}{2}\right]+2\left[\frac{g-1}{2}\right]^{2}\right)\right)\right) \\
& +O\left(|P|^{3 / 4}\left(\log _{q}|P|\right)^{2}\right) .
\end{aligned}
$$

As before, we deduce a corollary of Theorem 2.2 .

Corollary 2.2. Let $\mathbb{F}_{q}$ be a fixed finite field of odd cardinality $q$. Using the same notation as in the theorem we have,

$$
\sum_{P \in \mathcal{P}_{2 g+1, q}} L^{\prime \prime}\left(\frac{1}{2}, \chi_{P}\right) \sim \frac{1}{6} \log ^{2}(q)^{2}|P|\left(\log _{q}|P|\right)^{2}
$$

as $g \rightarrow \infty$.

Remark 2.3. Note that the average values are taken over the family of polynomials in $\mathcal{P}_{2 g+1}$, i.e., over odd degree monic irreducible polynomials. We could also consider the case of even degree polynomials, that is, averages over the family $\mathcal{P}_{2 g+2}$. In that case, the calculations are similar to the odd degree case and the only difference is the form of the approximate functional equation for $L\left(s, \chi_{P}\right)$ when $P \in \mathcal{P}_{2 g+2}$. For simplicity we only consider the odd degree case since the even degree case does not present any novelty. 
For a more detailed discussion about Dirichlet $L$-functions and Dirichlet characters in function fields we suggest the reader to consult [11, [4, Section 2] and [2, 7]. Throughout this paper we will let $|f|=q^{\operatorname{deg}(f)}$ be the norm of $f$.

The $L$-function associated to $\chi_{P}$ is the numerator of the zeta function associated to the hyperelliptic curve over $\mathbb{F}_{q}$ defined by the affine equation $C_{P}: y^{2}=P(T)$ and, consequently, $L\left(s, \chi_{P}\right)$ is a polynomial of degree $2 g$ in the variable $u=q^{-s}$ given by

$$
\begin{aligned}
L\left(s, \chi_{P}\right) & =\sum_{n=0}^{2 g} A\left(n, \chi_{P}\right) q^{-n s} \\
& =\sum_{n=0}^{2 g} \sum_{\substack{f \text { monic } \\
\operatorname{deg}(f)=n}} \chi_{P}(f) q^{-n s} .
\end{aligned}
$$

(see [11, Propositions 14.6 and 17.7] and [2, Section 3]).

It is well-known that this $L$-function satisfies a functional equation. Namely

$$
L\left(s, \chi_{P}\right)=\left(q^{1-2 s}\right)^{g} L\left(1-s, \chi_{P}\right),
$$

and the Riemann hypothesis for curves, proved by Weil [13], tell us that all the zeros of $L\left(s, \chi_{P}\right)$ have real part $1 / 2$.

Before we proceed with the proof of the two main results of this paper, we would like to point out that there is a different method that would also allow us to obtain the same results. By differentiating on both sides of the functional equation of $L\left(s, \chi_{P}\right)$, it should be possible to obtain the asymptotic formula of $L^{\prime}\left(\frac{1}{2}, \chi_{P}\right)$ using the results for the mean value of $L\left(\frac{1}{2}, \chi_{P}\right)$ from $[3$. And with a little more work and by using the mean value of $L\left(\frac{1}{2}, \chi_{P}\right)$ and $L^{\prime}\left(\frac{1}{2}, \chi_{P}\right)$ we could derive the mean value of $L^{\prime \prime}\left(\frac{1}{2}, \chi_{P}\right)$.

The approach described in the previous paragraph seems to work for arbitrary derivatives, although the full expansion as presented in Theorem 2.1 and 2.2 are not easy to derive by the use of this method. Because of that, the proofs of the main results of this paper are done by taking the derivative of the functional equation and averaging the character sums over monic irreducibles. In this way, we can keep track and see more clearly all the lower order terms.

In a forthcoming paper, we consider the second moment of higher derivatives of this family of $L$-functions and we also present conjectures for all the integral moments of derivatives of $L$-functions in function fields.

\section{The First Moment of $L^{\prime}\left(\frac{1}{2}, \chi_{P}\right)$}

From now on $P \in \mathcal{P}_{2 g+1, q}$. Changing $D$ for $P$ in the "approximate" functional equation [2, Lemma 3.3], we have that 


$$
L\left(s, \chi_{P}\right)=\sum_{\substack{f_{1} \text { monic } \\ \operatorname{deg}\left(f_{1}\right) \leq g}} \frac{\chi_{P}\left(f_{1}\right)}{\left|f_{1}\right|^{s}}+\left(q^{1-2 s}\right)^{g} \sum_{\substack{f_{2} \text { monic } \\ \operatorname{deg}\left(f_{2}\right) \leq g-1}} \frac{\chi_{P}\left(f_{2}\right)}{\left|f_{2}\right|^{1-s}} .
$$

The first derivative of the approximate functional equation (3.1) gives

$$
\begin{aligned}
L^{\prime}\left(\frac{1}{2}, \chi_{P}\right) & =-(\log (q)) \sum_{n=0}^{g} n q^{-n / 2} A\left(n, \chi_{P}\right) \\
& +(\log (q)) \sum_{m=0}^{g-1} A\left(m, \chi_{P}\right)(m-2 g) q^{-m / 2} \\
& =J_{1}+J_{2} .
\end{aligned}
$$

To prove theorem 2.1 we need to average 3.2 over $\mathcal{P}_{2 g+1, q}$. We will accomplish this task for each of the sums $J_{i}, i=1,2$ in (3.2). But before that, we need two results that will be used in the rest of the paper. The first one is the Polynomial Prime Theorem [11, Theorem 2.2] which states that

$$
\# \mathcal{P}_{2 g+1, q}=\frac{q^{2 g+1}}{2 g+1}+O\left(\frac{q^{g+\frac{1}{2}}}{2 g+1}\right) .
$$

The second result we need is a bound for a non-trivial character sum over function fields. Assume that $f$ is monic, $\operatorname{deg}(f)>0$ and that $f$ is not a perfect square. Rudnick has proved in [12] that

$$
\left|\sum_{\substack{P \text { monic } \\ \text { irreducible } \\ \operatorname{deg}(P)=n}}\left(\frac{f}{P}\right)\right| \ll \frac{\operatorname{deg}(f)}{n} q^{n / 2} .
$$

3.1. Averaging $J_{1}$ and $J_{2}$. We now proceed to prove an asymptotic formula for the average of $J_{1}$ and $J_{2}$. From equation (3.2) we split the character sum $A\left(n, \chi_{P}\right)$ when $f$ is a square of a polynomial and when $f$ is not a square of a polynomial and this gives us that

$$
\begin{aligned}
\sum_{P \in \mathcal{P}_{2 g+1, q}} J_{1} & =-(\log q) \sum_{n=0}^{g} n q^{-n / 2} \sum_{P \in \mathcal{P}_{2 g+1, q}} \sum_{\substack{\operatorname{deg}(f)=n \\
f=\square}} \chi_{P}(f) \\
& -(\log q) \sum_{n=0}^{g} n q^{-n / 2} \sum_{P \in \mathcal{P}_{2 g+1, q}} \sum_{\substack{\operatorname{deg}(f)=n \\
f \neq \square}} \chi_{P}(f) .
\end{aligned}
$$


Using the bound for non-trivial character sums (3.4) we have that the above is

$$
\begin{aligned}
\sum_{P \in \mathcal{P}_{2 g+1, q}} J_{1} & =-(\log q) \sum_{n=0}^{g} n q^{-n / 2} \sum_{P \in \mathcal{P}_{2 g+1, q}} \sum_{\substack{\operatorname{deg}(f)=n \\
f=\square}} \chi_{P}(f) \\
& +O\left(\sum_{n=0}^{g} n q^{-n / 2} \sum_{\operatorname{deg}(f)=n} \frac{q^{g} n}{2 g+1}\right)
\end{aligned}
$$

We have that the error term above is

$$
\begin{aligned}
& \sum_{n=0}^{g} n q^{-n / 2} \sum_{\operatorname{deg}(f)=n} \frac{q^{g} n}{2 g+1}=\sum_{n=0}^{g} n^{2} q^{n / 2} q^{g} \frac{1}{2 g+1} \\
& \ll q^{g} g^{2} q^{g / 2} \frac{1}{2 g+1} \\
& \ll|P|^{3 / 4}\left(\log _{q}|P|\right) .
\end{aligned}
$$

For the sum when $f$ is a perfect square we have that

$$
\begin{aligned}
& -(\log q) \sum_{n=0}^{g} n q^{-n / 2} \sum_{P \in \mathcal{P}_{2 g+1, q}} \sum_{\substack{\operatorname{deg}(f)=n \\
f=\square}} \chi_{P}(f) \\
& =-(\log q) \sum_{n=0}^{g} n q^{-n / 2} \sum_{\operatorname{deg}(l)=n / 2} \sum_{P \in \mathcal{P}_{2 g+1, q}} \chi_{P}\left(l^{2}\right) \\
& =-(\log q) \sum_{n=0}^{g} n q^{-n / 2} \sum_{\operatorname{deg}(l)=n / 2} \sum_{\substack{P \in \mathcal{P}_{2 g+1, q} \\
P \nmid l}} 1 .
\end{aligned}
$$

Using the Polynomial Prime Theorem (3.3), the fact that

$$
\sum_{\substack{P \in \mathcal{P}_{2 g+1, q} \\ P \nmid l}} 1=\sum_{P \in \mathcal{P}_{2 g+1, q}} 1
$$

since $\operatorname{deg}(P)>\operatorname{deg}(l)$, and after a few arithmetic manipulations and performing the sum over $n$ we obtain 


$$
\begin{aligned}
& -(\log q) \sum_{n=0}^{g} n q^{-n / 2} \sum_{P \in \mathcal{P}_{2 g+1, q}} \sum_{\substack{\operatorname{deg}(f)=n \\
f=\square}} \chi_{P}(f) \\
& =-(\log q) \frac{|P|}{\log _{q}|P|}\left[\frac{g}{2}\right]\left(\left[\frac{g}{2}\right]+1\right)+O\left(|P|^{1 / 2}\left(\log _{q}|P|\right)\right) .
\end{aligned}
$$

Combining (3.6) with the equation 3.10 we obtain

$$
\sum_{P \in \mathcal{P}_{2 g+1, q}} J_{1}=-(\log q) \frac{|P|}{\log _{q}|P|}\left[\frac{g}{2}\right]\left(\left[\frac{g}{2}\right]+1\right)+O\left(|P|^{3 / 4}\left(\log _{q}|P|\right)\right) .
$$

Since the average over $J_{2}$ is similar we shall not repeat the details. In the end, we obtain that

$$
\begin{aligned}
\sum_{P \in \mathcal{P}_{2 g+1, q}} J_{2} & =-2(\log q) \frac{|P|}{\log _{q}|P|} g\left(\left[\frac{g-1}{2}\right]+1\right) \\
& +(\log q) \frac{|P|}{\log _{q}|P|}\left[\frac{g-1}{2}+1\right]\left(\left[\frac{g-1}{2}\right]+1\right) \\
& +O\left(|P|^{3 / 4}\left(\log _{q}|P|\right)\right) .
\end{aligned}
$$

Putting together the average of the quantities $J_{1}$ and $J_{2}$ over $P \in \mathcal{P}_{2 g+1, q}$ allows us to deduce Theorem 2.1.

\section{The First Moment of $L^{\prime \prime}\left(\frac{1}{2}, \chi_{P}\right)$}

In this section we prove Theorem 2.2. The second derivative of the approximate functional equation (3.1) at $s=1 / 2$ gives

$$
\begin{aligned}
L^{\prime \prime}\left(\frac{1}{2}, \chi_{P}\right) & =(\log (q))^{2} \sum_{n=0}^{g} n^{2} q^{-n / 2} A\left(n, \chi_{P}\right) \\
& +(\log (q))^{2} \sum_{m=0}^{g-1} A\left(m, \chi_{P}\right)(m-2 g)^{2} q^{-m / 2} \\
& =S_{1}+S_{2},
\end{aligned}
$$

where

$$
A\left(n, \chi_{P}\right)=\sum_{\substack{f \text { monic } \\ \operatorname{deg}(f)=n}} \chi_{P}(f)
$$


4.1. Averaging $S_{1}$ and $S_{2}$. From equation (4.1) we have that

$$
\begin{aligned}
\sum_{P \in \mathcal{P}_{2 g+1, q}} S_{1} & =\sum_{P \in \mathcal{P}_{2 g+1, q}}(\log (q))^{2} \sum_{n=0}^{g} n^{2} q^{-n / 2} A\left(n, \chi_{P}\right) \\
& =\sum_{P \in \mathcal{P}_{2 g+1, q}}(\log (q))^{2} \sum_{n=0}^{g} n^{2} q^{-n / 2} \sum_{\substack{\operatorname{deg}(f)=n \\
f=\square}} \chi_{P}(f) \\
& +\sum_{P \in \mathcal{P}_{2 g+1, q}}(\log (q))^{2} \sum_{n=0}^{g} n^{2} q^{-n / 2} \sum_{\substack{\operatorname{deg}(f)=n \\
f \neq \square}} \chi_{P}(f) .
\end{aligned}
$$

For $f$ not a perfect square we use the bound given in equation (3.4) and the same reasoning used in 3.7 to write

$$
\begin{aligned}
& \sum_{P \in \mathcal{P}_{2 g+1, q}}(\log (q))^{2} \sum_{n=0}^{g} n^{2} q^{-n / 2} \sum_{\substack{\operatorname{deg}(f)=n \\
f \neq \square}} \chi_{P}(f) \\
& \ll \sum_{n=0}^{g} \sum_{\operatorname{deg}(f)=n} n^{2} q^{-n / 2} \frac{q^{g} n}{2 g+1} \\
& \ll|P|^{3 / 4}\left(\log _{q}|P|\right)^{2} .
\end{aligned}
$$

When $f$ is a perfect square we use the Prime Polynomial Theorem (3.3) to write

$$
\begin{aligned}
& \sum_{P \in \mathcal{P}_{2 g+1, q}}(\log (q))^{2} \sum_{n=0}^{g} n^{2} q^{-n / 2} \sum_{\substack{\operatorname{deg}(f)=n \\
f=\square}} \chi_{P}(f) \\
= & (\log (q))^{2} \sum_{n=0}^{g} n^{2} q^{-n / 2} \sum_{\substack{\operatorname{deg}(f)=n \\
f=l^{2}}} \sum_{P \in \mathcal{P}_{2 g+1, q}} \chi_{P}\left(l^{2}\right) \\
= & (\log (q))^{2} \sum_{n=0}^{[g / 2]} 4 m^{2} q^{-m} \sum_{\operatorname{deg}(l)=m}\left(\frac{q^{2 g+1}}{2 g+1}+O\left(\frac{q^{g}}{g}\right)\right) \\
= & \frac{2}{3}(\log q)^{2} \frac{|P|}{\log _{q}|P|}\left[\frac{g}{2}\right]\left(\left[\frac{g}{2}\right]+1\right)\left(2\left[\frac{g}{2}\right]+1\right)+O\left(q^{g} g^{2}\right) .
\end{aligned}
$$

Invoking equations 4.4 and 4.5 we proved that 


$$
\begin{aligned}
\sum_{P \in \mathcal{P}_{2 g+1, q}} S_{1} & =\frac{2}{3}(\log q)^{2} \frac{|P|}{\log _{q}|P|}\left[\frac{g}{2}\right]\left(\left[\frac{g}{2}\right]+1\right)\left(2\left[\frac{g}{2}\right]+1\right) \\
& +O\left(|P|^{3 / 4}\left(\log _{q}|P|\right)^{2}\right) .
\end{aligned}
$$

Using the Prime Polynomial Theorem (3.3), the bound for non-trivial character sums (3.4) and equation (4.1) a similar argument can be used to establish

$$
\begin{aligned}
\sum_{P \in \mathcal{P}_{2 g+1, q}} S_{2} & =\frac{2}{3}(\log q)^{2} \frac{|P|}{\log _{q}|P|}\left(\left(\left[\frac{g-1}{2}\right]+1\right)\right. \\
& \left.\times\left(6 g^{2}+\left[\frac{g-1}{2}\right]-6 g\left[\frac{g-1}{2}\right]+2\left[\frac{g-1}{2}\right]^{2}\right)\right) \\
& +O\left(|P|^{3 / 4}\left(\log _{q}|P|\right)^{2}\right) .
\end{aligned}
$$

Combining equations (4.6) and (4.7) we establish Theorem 2.2.

Acknowledgement. We would like to express our gratitude to an anonymous referee whose comments improved the presentation and clarity of the paper.

\section{REFERENCES}

[1] Andrade, J.C., Jung H.: Mean values of derivatives of $L$-functions in function fields: II, (submitted).

[2] Andrade, J.C., Keating, J.P.: The mean value of $L\left(\frac{1}{2}, \chi\right)$ in the hyperelliptic ensemble, J. Number Theory, 132, 2793-2816 (2012).

[3] Andrade, J.C., Keating, J.P.: Mean Value Theorems for $L$-functions over prime polynomials for the rational function field, Acta Arith., 161, 371-385 (2013).

[4] Andrade, J.C., Rajagopal S.: Mean values of derivatives of $L$-functions in function fields: I, J. Math. Anal. Appl., 443, 526-541 (2016).

[5] Conrey, B.: The Fourth Moment of Derivatives of the Riemann zeta-function, Q. J. Math., 39, 21-36 (1988).

[6] Conrey, B., Rubinstein, M., Snaith, N.: Moments of the Derivative of Characteristic Polynomials with an Application to the Riemann Zeta Function, Commun. Math. Phys., 267, 611-629 (2006).

[7] Faifman, D., Rudnick, Z.: Statistics of the zeros of zeta functions in families of hyperelliptic curves over a finite field, Compos. Math., 146, 81-101 (2010).

[8] Gonek, S.: Mean values of the Riemann zeta-function and its derivatives, Invent. Math., 75, 123-141 (1984).

[9] Hoffstein, J., Rosen, M.: Average values of $L$-series in function fields, J. Reine Angew. Math., 426, 117-150 (1992).

[10] Ingham, A.E.: Mean-value theorems in the theory of the Riemann zeta-function, Proc. Lond. Math. Soc., 27, 273-300 (1926).

[11] Rosen, M.: Number Theory in Function Fields. Graduate Texts in Mathematics vol. 210. Springer-Verlag, New York (2002). 
[12] Rudnick, Z.: Traces of high powers of the Frobenius class in the hyperelliptic ensemble, Acta Arith., 143, 81-99 (2010).

[13] Weil, A.: Sur les Courbes Algébriques et les Variétés qui s'en Déduisent. Hermann, Paris (1948).

Department of Mathematics, University of Exeter, Exeter, EX4 4QF, United KINGDOM

E-mail address: j.c.andrade@exeter.ac.uk 